\title{
The Meijer's G-functions: Convenient for describing $\beta$ decay in unstable nuclei
}

\author{
A. Pishkoo \\ Physics and Accelerators Research School, NSTRI, Tehran, Iran \\ apishkoo@gmail.com
}

Keywords: Meijer's G-function; $\beta$ decay; Special functions; Gamma function.

Abstract: The purpose of this paper is to show that the Meijer's G-functions which include all elementary and most of the special functions can describe some phenomena in nuclear physics. In fact, some interesting properties of Meijer's G-functions triggered us to apply these functions in the process $\beta$ decay. These functions act as "wave functions" for parent and daughter nuclei.

\section{INTRODUCTION}

Perhaps, Cornelis Simon Meijer (1936) was the first to introduce the G-function in mathematics. This very general function intended to include most of the known special functions as particular cases and for a long time, many studies have been done involving this type of functions. These functions have a lot of practical applications in the fields of mathematical physics, theoretical physics, mathematical analysis, etc. [1]. Recently Pishkoo and Darus obtain G-function solutions for Reaction-diffusion equation [2], Schrodinger equation [3], Diffusion equation, and Laplace's equation $[4,5]$, respectively.

In [6] some properties of Meijer's G-function are applied for obtaining excited states (radial functions) of the Hydrogen atom from ground state. Our results are summarized in Table 1. As it is shown in Table 1, all radial states of the Hydrogen atom belong to the family of functions $\boldsymbol{G}_{\mathbf{0}, \mathbf{1}}^{\mathbf{1 , 0}}$ which are in general sum of $\boldsymbol{G}_{\mathbf{0}, \mathbf{1}}^{\mathbf{1 , 0}} \mathrm{s}^{\prime}$ with different parameters.

All of these states are shown by Meijer's G-function. Because many obtaining results from atomic physics can be generalized to nuclear physics, here we assume that the wave functions of parent and daughter nuclei are of type Meijer's G-function, and then we describe $\beta$ decay.

TABLE 1

Derivation of excited radial states of the Hydrogen atom from the ground state $R_{10}$ in terms of Meijer's

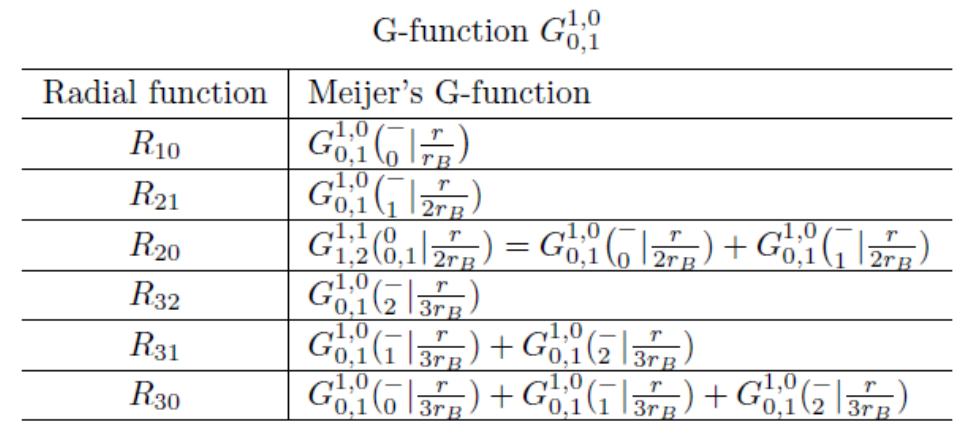

\section{MEIJER'S G-FUNCTIONS}

We begin with the definition of Meijer's G-function as the following:

Definition 2.1. A definition of the Meijer's G-function is given by the following path integral in the complex plane, called Mellin-Barnes type integral [1,6-9]:

$$
G_{p, q}^{m, n}\left(p_{1}, \ldots, b_{q} \mid a_{1}, \ldots\right)=\frac{1}{2 \pi i} \int_{L} \frac{\prod_{j=1}^{m} \Gamma\left(b_{j}-s\right) \prod_{j=1}^{n} \Gamma\left(1-a_{j}+s\right)}{\prod_{j=m+1}^{q} \Gamma\left(1-b_{j}+s\right) \prod_{j=n+1}^{p} \Gamma\left(a_{j}-s\right)} z^{s} d s .
$$


Here, the integers $m ; n ; p ; q$ are called "orders" of the G-function, or the components of the order $(m ; n ; p ; q) ; a_{j}$ and $b_{j}$ are called "parameters" and in general, they are complex numbers. The definition holds under the following assumptions: $0 \leq m \leq q$ and $0 \leq n \leq p$, where $m$; $n$; $p$; and $q$ are integer numbers. Subtracting parameters $a_{j}-b_{k} \neq 1,2, \ldots$ for $k=1, \ldots, n$ and $j=1,2, \ldots, m$ imply that no pole of any $\Gamma\left(b_{j}-s\right), j=1, \ldots, m$ coincides with any pole of any $\Gamma\left(1-a_{k}+s\right), k=$ $1, \ldots, n$.

Choosing $m=1 ; n=0 ; p=0$ and $q=1$, we have

$$
G_{0,1}^{1,0}\left(-\overline{b_{1}} \mid z\right)=\frac{1}{2 \pi i} \int_{L} \Gamma\left(b_{1}-s\right) z^{s} d s
$$

Or in more general in terms of elementary function is (see [1])

$$
G_{0,1}^{1,0}\left(\frac{{ }_{\alpha}}{\alpha} \mid \eta z^{\alpha}\right)=\eta^{\frac{\beta}{\alpha}} z^{\beta} e^{-\eta z^{\alpha}}
$$

Based on the definition, the following basic properties are easily derived:

$$
z^{\alpha} G_{p, q}^{m, n}\left(\begin{array}{c}
\mathbf{a}_{\mathbf{p}} \\
\mathbf{b}_{\mathbf{q}}
\end{array}\right)=G_{p, q}^{m, n}\left(\begin{array}{c}
\mathbf{a}_{\mathbf{p}}+\alpha \\
\mathbf{b}_{\mathbf{q}}+\alpha
\end{array}\right),
$$

where the multiplying term $z^{\alpha}$ changes the parameters of the G-function. The derivatives of arbitrary order $k$ can change the G-function's orders and parameters:

$$
z^{k} \frac{d^{k}}{d z^{k}} G_{p, q}^{m, n}\left(\begin{array}{c}
\mathbf{a}_{\mathbf{q}} \\
\mathbf{b}_{\mathbf{q}}
\end{array}\right)=G_{p+1, q+1}^{m, n+1}\left(\begin{array}{c}
\mathbf{0}, \mathbf{a}_{\mathbf{p}}, \mathbf{k} \\
\mathbf{b}_{\mathbf{p}}
\end{array}\right)
$$

The G-function is symmetric in the groups of parameters which are separated:

$\left(a_{1}, \ldots, a_{n}\right) ;\left(b_{1}, \ldots, b_{m}\right) ;\left(a_{n+1}, \ldots, a_{p}\right) ;\left(b_{m+1}, \ldots, b_{q}\right)$. If one of the $a_{j}^{\prime} s j=1, \ldots, n$; is equal to some of the $b_{k}^{\prime} s k=m+1, \ldots, q$; then the G-function reduces to one of Lower order. For example, if $n, p, q \geq 1$

$$
G_{p, q}^{m, n}\left(\begin{array}{l}
a_{1}, \ldots, a_{p} \\
b_{1}, \ldots, b_{q-1}, a_{1}
\end{array} \mid z\right)=G_{p-1, q-1}^{m, n-1}\left(\begin{array}{l}
a_{2}, \ldots, a_{p} \\
b_{1}, \ldots, b_{q-1}
\end{array} \mid z\right) .
$$

Similarly, if one of the $a_{j}^{\prime} s, j=n+1, \ldots, p$; is equal to some of the $b_{k}, k=1, \ldots, m$; the function has its orders $m ; p$; $q$ reduced by 1 , for example, if $m, p, q \geq 1$;

$$
G_{p, q}^{m, n}\left(\begin{array}{c}
a_{1}, \ldots, a_{p-1}, b_{1} \\
b_{1}, b_{2}, \ldots, b_{q}
\end{array} \mid z\right)=G_{p-1, q-1}^{m-1, n}\left(\begin{array}{c}
a_{1}, \ldots, a_{p-1}, \ldots, b_{q} \\
\left.b_{2}, z\right)
\end{array}\right.
$$

\section{BETA DECAY}

In $\beta$-decay the charge of a nucleus changes while $\mathbf{A}$ remains fixed. This process occurs either by the simultaneous emission of an electron and an anti-neutrino, or a positron and a neutrino, or by the capture of an atomic electron with the emission of a neutrino [11-12]. As an example, ${ }_{32}^{77} \mathrm{Ge}$ decays by a series of $\beta$-decays to ${ }_{34}^{77} S e, \mathbf{Z}$ increasing by one at each stage: 


$$
\begin{aligned}
{ }_{32}^{77} \mathrm{Ge} \rightarrow & \rightarrow{ }_{33}^{77} \mathrm{As}+e^{-}+\bar{\nu}_{e}+2.75 \mathrm{Mev} \\
& \downarrow \\
& { }_{34}^{77} \mathrm{Se}+e^{-}+\bar{\nu}_{e}+0.68 \mathrm{Mev} .
\end{aligned}
$$

${ }_{36}^{77} \mathrm{Kr}$ decay by emitting a positron and a neutrino. Another sequence of decays ending in ${ }_{34}^{77} \mathrm{Se}$ is:

$$
\begin{aligned}
{ }_{36}^{77} \mathrm{Kr} \rightarrow & \rightarrow{ }_{35}^{77} \mathrm{Br}+e^{+}+\nu_{e}+2.89 \mathrm{Mev} \\
& \downarrow \\
& { }_{34}^{77} \mathrm{Se}+e^{+}+\nu_{e}+1.36 \mathrm{Mev} .
\end{aligned}
$$

\section{MAIN RESULTS}

In order to use the Meijer's G-functions as the convenient language in nuclear physics we give our idea in the format of four postulates as follows:

1-Each nuclear (or atomic) state can be represented by a Meijer's G-function, $G_{p, q}^{m, n}\left(\begin{array}{c}a_{p} \\ b_{q}\end{array} \mid z\right)$ which the values of all orders and parameters determine exactly the one nuclear (or atomic) state.

If each G-function specifies a nuclear state, then the transitions can be expressed by using properties of G-functions. The equations (4), (5), (6) and (7) associate G-functions together.

2- According to (4) when the operator $\mathrm{z}$ is multiplied by $G_{p, q}^{m, n}\left({ }_{b_{q}}^{a_{p}} \mid z\right)$ it acts as $\gamma$ photon that gives energy to electron to shift from the initial state (lower energy) to the final state (higher energy) which gives another

Meijer's G-function $G_{p, q}^{m, n}\left(\begin{array}{l}a_{p}+\alpha \\ b_{q}+\alpha\end{array} \mid z\right)$.

$$
z G_{p, q}^{m, n}\left(\begin{array}{l}
\mathbf{b}_{\mathbf{p}} \\
\mathbf{b}_{\mathbf{q}}
\end{array}\right)=G_{p, q}^{m, n}\left(\begin{array}{l}
\mathbf{b}_{\mathbf{p}}+1 \\
\mathbf{b}_{\mathbf{q}}+1
\end{array} \mid z\right) .
$$

Vice versa, if we use the following notation and interpretation:

$$
G_{p, q}^{m, n}\left(\begin{array}{c}
\mathbf{a}_{\mathbf{p}}+1 \\
\mathbf{b}_{\mathbf{q}}+1
\end{array} \mid z\right)=G_{p, q}^{m, n}\left(\begin{array}{c}
\mathbf{b}_{\mathbf{p}} \\
\mathbf{b}_{\mathbf{q}}
\end{array}\right) z .
$$

It acts as $\gamma$ photon that exit form atom because of transition from upper state to lower state. Note that an interaction of $\gamma$ rays with the matter is of type "atomic" interaction not "nuclear".

3- According to (5) when the operator $z \frac{d}{d z}$ is multiplied by $G_{p, q}^{m, n}\left({ }_{b_{q}}^{a_{p}} \mid z\right)$ it acts as $\gamma$ photon that gives energy to electron to shift from the initial state (lower energy) to the final state (high energy) which gives another Meijer's G-function $G_{p, q}^{m, n}\left({ }_{b_{q}, 1}^{0, a_{p}} \mid z\right)$. Note again that interaction of $\gamma$ rays with the matter is of type "atomic" interaction not "nuclear".

4- According to (6) when the G-function is symmetric in the groups of parameters, then $G_{p, q}^{m, n}\left({ }_{b_{1}, \ldots, b_{q-1}, a_{1}} a_{1}, \ldots, a_{p}\right)$ can describe the nuclear excited state before $\gamma$ or $\beta$ decay, and $G_{p-1, q-1}^{m, n-1}\left(\begin{array}{c}a_{2}, \ldots, a_{p} \\ b_{1}, \ldots, b_{q-1}\end{array} \mid z\right)$ gives the lower energy (excited or ground)nuclear state. For example suppose that we want to express beta decays of nuclei $\left({ }_{32}^{77} \mathrm{Ge}\right.$ to ${ }_{33}^{77} \mathrm{As}$ and ${ }_{33}^{77} \mathrm{As}$ to $\left.{ }_{34}^{77} \mathrm{Se}\right)$ \& $\left({ }_{36}^{77} \mathrm{Kr}\right.$ to ${ }_{35}^{77} \mathrm{Br}$ and ${ }_{35}^{77} \mathrm{Br}$ to ${ }_{34}^{77} \mathrm{Se}$ ) in terms of G-functions. Although we do not know which Gfunction exactly describes each nucleus, assume the following example: 
Using equation (2.5) two times gives

$$
G_{4,4}^{2,3} \rightarrow G_{3,3}^{2,2} \rightarrow G_{2,2}^{2,1}
$$

and may represent $\beta$ decay by emitting electron, while using equation (2.6) two times gives

$$
G_{4,4}^{4,1} \rightarrow G_{3,3}^{3,1} \rightarrow G_{2,2}^{2,1}
$$

and may represent $\beta$ decay by emitting positron.

These postulates are starting point and building blocks for giving new describing version of nuclear physics, here for $\beta$ decay.

\section{REFERENCES}

[1] V. Kiryakova, "Generalized Fractional Calculus and Applications", Longman, Harlow, UK, 1994.

[2] A. Pishkoo, M. Darus, "Meijer's G-functions (MGFs) in Micro- and Nano-structures". Journal of Computational and Theoretical Nanoscience, vol. 10, pp. 2478-2483, October, 2013.

[3] A. Pishkoo, M. Darus, "G-function Solutions for Schrodinger Equation in Cylindrical Coordinates System". Journal of Applied mathematics, vol. 5, pp. 342-346, February, 2014.

[4] A. Pishkoo, M. Darus, "Some Applications of Meijer G-functions as Solutions of Differential Equations in Physical Models", Journal of Mathematical Physics, Analysis, Geometry, vol. 3, issue 3, pp. 379-391, 2013.

[5] A. Pishkoo, M. Darus, "G-function Solutions for Diffusion and Laplace's Equations", Journal of Advances in Mathematics, Vol. 4, issue 1, pp. 359-365, 2013.

[6] A. Pishkoo, "The simplest Meijer's G-function $\boldsymbol{G}_{\mathbf{0}, \mathbf{1}}^{\mathbf{1 , 0}}$ as the radial functions of the Hydrogen atom", Bulletin of Society for Mathematical Services \& Standard, Vol. 3, No. 2, pp. 1-5, 2014.

[7] L. Andrews, "Special functions for engineers and applied mathematicians", Macmillan, 1985.

[8] Y. Luck, "The Special Functions and Their Approximations, Complex Variables", New York: Academic press, 1969.

[9] R. Askey, "Meijer G-function", Cambridge: Cambridge University Press, 2010.

[10] A. Klimyik, A. "Meijer G-function", Berlin: Springer, 2001.

[11] W. N. Cottingham, D. A. Greenwood, "An introduction to Nuclear Physics", Cambridge University Press, 2001.

[12] S. G. Prussin, "Nuclear Physics for Applications", Willey-VCH, 2007. 